\title{
E. Enseñar derechos humanos en la educación. Experiencias durante la pandemia
}

\author{
Teaching human rights in higher \\ education. Experiences during the \\ pandemic
}

\section{Ensinar direitos humanos no ensino superior. Experiências durante a pandemia}

\author{
Victoria Kandel ${ }^{1}$ \\ Natalia $\mathrm{Naz}^{2}$ \\ Andrea Vallejos ${ }^{3}$
}

\section{Resumen}

A lo largo de estas páginas nos proponemos analizar la vivencia de un espacio educativo concreto y acotado: nos referimos a los modos en que estudiantes de la Universidad Nacional de Lanús, en la Argentina, han atravesado su experiencia educativa durante el año 2020, el año de la pandemia. Presentamos los resultados de una encuesta realizada durante el año 2020 a 537 estudiantes, los cuales respondieron a un cuestionario autosuministrado de manera virtual en los meses de julio y de noviembre. El relevamiento buscó conocer sus percepciones sobre la formación en derechos humanos que reciben durante su segundo año de estudios. A partir de

Recibido: 1-7-2021 - Aceptado: 5-10-2021

1 Licenciada en Ciencia política y Doctora en Educación. Profesora e investigadora en el Instituto de Justicia y Derechos Humanos de la Universidad Nacional de Lanús, Argentina. Afiliación institucional: Instituto de Justicia y Derechos Humanos "Eduardo Luis Duhalde" Secretaría de Ciencia y Técnica-Universidad Nacional de Lanús, Argentina. Correo electrónico: kandelv@gmail.com(iD https://orcid.org/0000-0002-3629-4739

2 Técnica Nacional en Tiempo Libre y Recreación; Licenciada en Trabajo Social, Doctoranda en Derechos Humanos. Becaria doctoral cofinanciada UNLa - CONICET. Afiliación institucional: Instituto de Justicia y Derechos Humanos "Eduardo Luis Duhalde"-Secretaría de Ciencia y Técnica-Universidad Nacional de Lanús, Argentina. Correo electrónico: nataliagnaz@gmail.com (D) https://orcid.org/0000-0003-1651-4885

3 Licenciada en Trabajo Social, Especialista en Políticas Públicas y Género (CLACSO/ FLACSO BRASIL), Maestranda en DDHH (UNLa, Argentina), Doctoranda en Ciencias Sociales (Universidad de General Sarmiento- IDES, Argentina). Docente adjunta en el Seminario de Justicia y DDHH UNLa. Afiliación institucional: Departamento de Planificación y Políticas Públicas, Universidad Nacional de Lanús, Argentina. Correo electrónico: vallejos.ab@gmail.com (iD) https://orcid.org/0000-0002-4744-6806 
una resolución del Consejo Superior, todas las personas que estudian en dicha universidad deben realizar un curso sobre derechos humanos, con lo que se busca avanzar hacia la formación de profesionales que se sientan inmersos e interpelados por una cultura de los derechos humanos. Por lo tanto, en este artículo nos proponemos dar cuenta de dos cuestiones a la vez: conocer los modos en que los estudiantes perciben y otorgan significados a los derechos humanos, y conocer cuál ha sido su experiencia de formación en derechos humanos en los meses de pandemia.

Palabras clave: universidades, derechos humanos, enseñanza, pandemia.

\begin{abstract}
Throughout these pages we propose to analyze the experience of a specific and limited educational space: we refer to the ways in which students at the Universidad Nacional de Lanús, in Argentina, have gone through their educational experience during 2020, the year of the pandemic. We present the results of a survey conducted during 2020 to 537 students, who responded to a virtual self-administered questionnaire in July and November. The survey sought to know their perceptions about the training in human rights that they receive during their second year of studies. Based on a resolution of the Superior Council, all the people who study at said university must take a course on human rights, which seeks to advance towards the training of professionals who feel immersed and challenged by a culture of human rights. Therefore, in this article we propose to address two issues at the same time: to know the ways in which students perceive and give meaning to human rights, and to know what their experience of training in human rights has been in the months of pandemic.
\end{abstract}

Keywords: universities, human rights, teaching, pandemic.

\title{
Resumo
}

Ao longo destas páginas nos propomos a analisar a experiência de um espaço educacional específico e limitado: nos referimos às maneiras pelas quais os alunos da Universidad Nacional de Lanús, na Argentina, viveram sua experiência educacional durante o ano de 2020, ano da pandemia. Apresentamos os resultados de uma pesquisa realizada durante o ano de 2020 junto a 537 alunos, que responderam a um questionário virtual autoaplicável nos meses de julho e novembro. A pesquisa buscou conhecer suas percepções sobre a formação em direitos humanos que recebem durante o segundo ano de estudos. Com base em uma resolução do Conselho Superior, todas as pessoas que estudam na referida universidade devem fazer um curso de direitos humanos, que visa avançar na formação de profissionais que se sintam imersos e desafiados por uma cultura de direitos humanos. Portanto, neste artigo nos propomos a abordar duas questões ao mesmo tempo: conhecer as formas como os alunos percebem e dar sentido aos direitos humanos, e saber como tem sido sua experiência de formação em direitos humanos nos meses de pandemia.

Palavras-chave: universidades, direitos humanos, ensino, pandemia. 


\section{Presentación}

El año 2020 arrasó con nuestra cotidianeidad, nuestros modos de organizar la vida social, cultural, el trabajo y los vínculos. Esta irrupción tuvo, y continúa teniendo, consecuencias en la vida de quienes habitamos este planeta, debiendo transitar estas vivencias de manera colectiva, compartiendo angustias e incertidumbres con la Humanidad toda.

En este marco, nos proponemos analizar a continuación la vivencia de un espacio educativo concreto y acotado: nos referimos a los modos en que estudiantes de la Universidad Nacional de Lanús, en la Argentina, han atravesado su experiencia educativa durante el año 2020, el año de la pandemia.

El trabajo que se presenta analiza las respuestas que ofreció un grupo de estudiantes que ha cursado el Seminario de Justicia y Derechos Humanos. Se trata de una asignatura obligatoria para quienes estudian en esta casa de altos estudios, por lo que nos proponemos analizar dos ejes: 1) la vivencia de quienes estudiaron en la Universidad Nacional de Lanús en tiempos de pandemia. 2) sus percepciones sobre el aprendizaje de los derechos humanos.

Nos preguntamos: ¿Por qué estudiar derechos humanos en la universidad? ¿Cómo vivieron y qué aprendieron durante el 2020 , en su rol de estudiantes-en-pandemia? ¿Qué saben sobre los derechos humanos? ¿Cómo impactan estos aprendizajes en los modos de pensar a los derechos humanos y a su profesión?

\section{Introducción. Formación en derechos humanos en el nivel universitario}

Si a nivel internacional existen acuerdos sobre la importancia de la Educación en Derechos Humanos (EDH) como herramienta que permite avanzar hacia una cultura de derechos humanos, dicho consenso también halla una expresión y un anclaje particular en el caso argentino. Podemos recurrir a la definición de EDH que ofrece el Programa Mundial para la Educación en Derechos Humanos de las Naciones Unidas:

La educación en la esfera de los derechos humanos es un proceso a largo plazo que se prolonga durante toda la vida, en el cual todas las personas aprenden a ser tolerantes, a respetar la dignidad de los demás y los medios y arbitrios de asegurar ese respeto en todas las sociedades.

La educación en la esfera de los derechos humanos es esencial para la realización de los derechos humanos y las libertades fundamentales y contribuye significativamente a promover la igualdad, prevenir los conflictos y las violaciones de los 
derechos humanos y fomentar la participación y los procesos democráticos, a fin de establecer sociedades en que se valore y respete a todos los seres humanos, sin discriminaciones ni distinciones de ningún tipo, en particular por motivos de raza, color, sexo, idioma, religión, opinión política o de otra índole, origen nacional o social, posición económica, nacimiento o cualquier otra condición (Asamblea General ONU, Resolución 59/113, 2004: Párr. 5-6).

La EDH encuentra modos singulares de desarrollo en los contextos y geografías particulares. En ese sentido, y anclándonos en el caso de la educación superior argentina, los derechos humanos se encuentran presentes de diversos modos y en variadas formas institucionales desde el retorno de la democracia en 1983.

Desde la creación de cátedras abiertas y libres durante los años ochenta, en la década del noventa el surgimiento de carreras de posgrado y más cerca en el tiempo, surgieron ámbitos institucionales de derechos humanos bajo la figura de Centros, Institutos, Observatorios, Secretarías o Subsecretarías. En los últimos años algunas universidades están dando importantes pasos para incluir en sus currículas contenidos en derechos humanos, pues una educación superior de calidad no debe desentenderse de promover una reflexión sobre el sentido social de la profesión y las complejidades del contexto en el cual los futuros profesionales habrán de desempeñarse: de ahí el compromiso con la curricularización de contenidos en derechos humanos.

\section{Enseñanza de derechos humanos en la Universidad Nacional de Lanús}

A partir del año 2014 quienes cursan licenciaturas y ciclos complementarios en la Universidad Nacional de Lanús deben cursar una asignatura titulada "Seminario de Justicia y Derechos Humanos". Junto a otros cursos cuatrimestrales transversales y obligatorios (inglés, informática y una asignatura denominada "Pensamiento Nacional") este espacio curricular pretende interpelar al conjunto de quienes cursan una carrera universitaria.

El carácter transversal del Seminario habilita la posibilidad de encuentro, diálogo y reflexión de distintas disciplinas con relación a campos problemáticos, lo que se inscribe en la propuesta general de la universidad que se define como una organización académica basada en problemas sociales antes que en disciplinas aisladas.

Dado este carácter transversal en este artículo, nos proponemos compartir reflexiones derivadas de la instancia de devolución que quienes realizan este curso completan al concluir el mismo. Dicha instancia se realiza mediante la aplicación de una encuesta anónima de carácter optativa y virtual. 
Los datos analizados en el presente artículo corresponden a la encuesta implementada durante el primer y segundo cuatrimestre del año 2020. En este relevamiento nos propusimos analizar la percepción de quienes estudian en la Universidad Nacional de Lanús respecto a los derechos humanos en general, y a la experiencia de haber aprendido sobre esta temática en particular. Dado el carácter excepcional en que se cursó durante el 2020, la encuesta buscó relevar también su experiencia universitaria cursando de manera enteramente virtual.

La asignatura tiene una duración de cuatro meses, y está planteada para ser cursada una vez a la semana, con una carga horaria total de $64 \mathrm{hs}$. Quienes cursan derechos humanos se encuentran promediando su segundo año en sus respectivas carreras. Fueron analizadas 537 encuestas: 378 mujeres, 156 varones, 2 se estudiantes se identifican géneros no binarios y un estudiante varón trans. La mayoría de las personas encuestadas tiene entre 17 y 27 años (321 casos), pero la edad promedio es de 42 años. Esto es así porque en la UNLa, y en el país en general, se observa una presencia cada vez mayor de personas adultas en las aulas universitarias.

Tengamos en cuenta que la Universidad Nacional de Lanús se encuentra ubicada en una zona periférica del Conurbano de Buenos Aires, y atiende a una población de características socioeconómicas desfavorables. Al igual que otras universidades públicas inauguradas en los últimos treinta años, se trata de una institución que alberga a estudiantes que en su mayoría son primera generación de estudiantes universitarios. La mayoría trabaja mientras estudia y su promedio de edad es mayor que el de las universidades tradicionales.

Recorriendo la UNLa, es posible observar que la misma cuenta con una oferta de 19 carreras de pregrado (Tecnicaturas y títulos intermedios), 24 carreras de grado y 20 de posgrado, aunque el relevamiento que presentamos en este artículo no contempla las carreras de posgrado. Las carreras están agrupadas en 4 departamentos: Desarrollo productivo y tecnológico (tiene 16 carreras), Humanidades y Arte (tiene 8 carreras), Planificación y Políticas Públicas (12 carreras) y Salud Comunitaria (7 carreras). Conviene aclarar que el Departamento de Desarrollo productivo tiene una matrícula de 6928 estudiantes en grado y pregrado. El Departamento de Humanidades y Artes tiene 4351 estudiantes, el Departamento de Planificación y Políticas Públicas tiene 3703 estudiantes, mientras que el de Salud Comunitaria tiene 6909. La matrícula de carreras de grado y pregrado suma en total 21.891.

Podemos observar la siguiente distribución en los cuatro Departamentos de la UNLa: 


\section{Tabla 1}

Respuestas según Departamento donde se estudia

\begin{tabular}{|l|l|l|}
\hline \multicolumn{1}{|c|}{ Departamento } & \multicolumn{1}{c|}{ Respuestas } & \multicolumn{1}{c|}{ Matrícula total } \\
\hline Total Humanidades y Artes & $106(19.7 \%)$ & $4351(19.9 \%)$ \\
\hline Total Planificación y Políticas Públicas & $103(19.2 \%)$ & $3703(16.9 \%)$ \\
\hline Total Desarrollo Productivo y Tecnológico & $133(24.8 \%)$ & $6928(31.6 \%)$ \\
\hline Total Salud Comunitaria & $189(35.2) \%$ & $6909(31.6 \%)$ \\
\hline No responde & $6(1.1 \%)$ & \\
\hline Total general & $537(100 \%)$ & $21981(100 \%)$ \\
\hline
\end{tabular}

\section{Pandemia, “ASPO” y desafíos}

La Pandemia por Covid-19 enfrentó al sistema educativo en general y a las Universidades Nacionales con la necesidad de reorganización y adaptación a las características del contexto.

En el caso de la Argentina el gobierno nacional dispuso el "aislamiento social, preventivo y obligatorio" (ASPO), por medio del decreto 297/2020, lo cual implicó que mientras estuvo vigente dicha normativa las personas debían permanecer en sus domicilios habituales, solo pudiendo realizar desplazamientos mínimos e indispensables para aprovisionarse de artículos de limpieza, medicamentos y alimentos (República Argentina, Decreto 297/2020, Art. 2)

En virtud de esta medida en el caso de la UNLa dispuso la suspensión de las clases presenciales en marzo del 2020 y la continuidad del cuatrimestre mediante el campus virtual, situación que se extendió durante todo el año 2020 y que persiste al promediar el 2021. Se organizaron capacitaciones virtuales tanto para docentes como para estudiantes.

La continuidad pedagógica fue entonces garantizada por la virtualización de todo el sistema educativo, a través de distintas estrategias y soportes. En el caso del sistema de educación superior, y particularmente del subsistema universitario, se debió pensar en una universidad "desterritorializada", acogiendo diversas demandas y necesidades de aprendizaje por parte de las y los estudiantes.

La problemática de la "brecha digital" sin bien no es novedosa, se agudizó durante este año, generando circuitos de enseñanza y aprendizaje diferenciados según las posibilidades de acceso a internet y las condiciones en que las personas que estudian 
pudieron realizar sus estudios universitarios. Tal como lo señaló un informe del Instituto Internacional de la UNESCO para la Educación Superior en América Latina y el Caribe en 2020: "Independientemente del nivel de educación, el peligro primordial es que las desigualdades en el aprendizaje se amplíen, aumente la marginación y los estudiantes más desfavorecidos se vean imposibilitados de proseguir sus estudios" (UNESCO IESALC, 2020, p. 5).

Esta cuestión fue fuertemente discutida en el país, al tiempo que se realizaron distintos relevamientos para conocer la situación de conectividad en diferentes regiones y sectores sociales de nuestro territorio. En ese marco, (y por mencionar solo uno de esos estudios) el Ente Nacional de Comunicaciones junto con el Consejo Provincial de Coordinación del Sistema Universitario y Científico realizaron un relevamiento para conocer la situación específica de estudiantes de universidades situadas en la Provincia de Buenos Aires. El estudio revela que los principales problemas consignados para dar continuidad a sus estudios fueron dados por las dificultades en la conectividad y falta de equipamiento tecnológico para acceder a las clases. Además, cerca del 14\% declaró no contar con un servicio de wifi para acceder a internet. $\mathrm{Y}$ del alto porcentaje que sí tiene wifi, una gran cantidad valora al servicio como regular o malo.

\section{Enseñar y aprender derechos humanos en tiempos de pandemia}

En líneas generales, la asignatura les resultó entre muy buena (51\%) y buena (39\%). Y al preguntarles cómo les resultó la experiencia de haber cursado en la modalidad virtual, las respuestas fueron las que se muestran en el gráfico número 1. 


\section{Gráfico 1}

¿Cómo calificarías tu experiencia en la modalidad virtual?

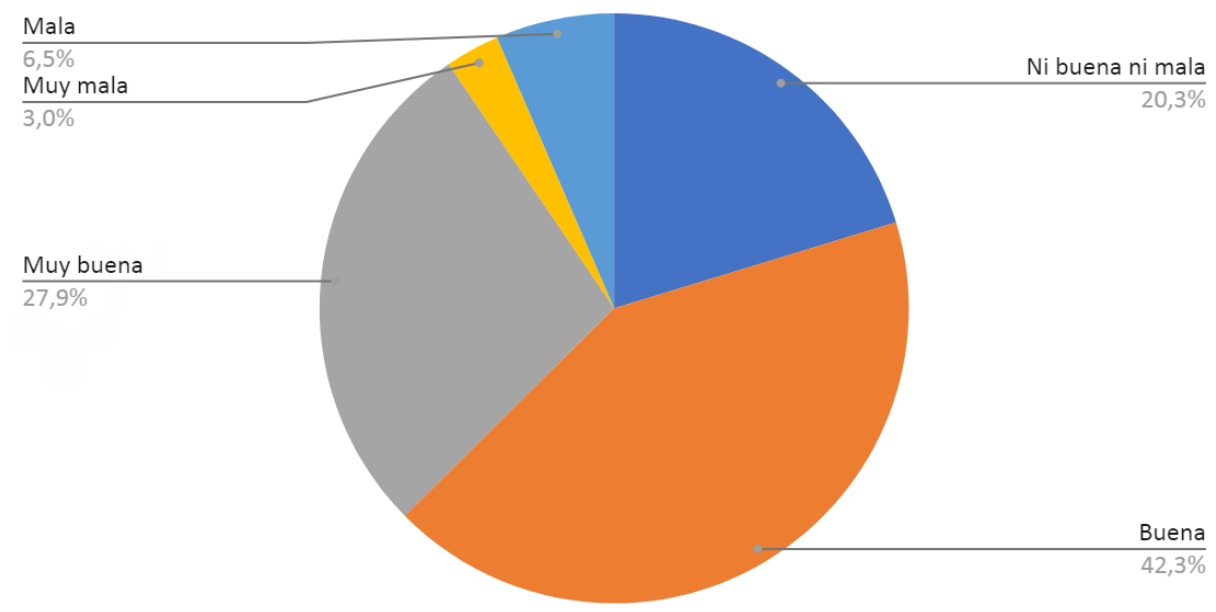

Nota: elaboración propia en base a los resultados de la encuesta realizada durante el año 2020 a 537 estudiantes.

Es decir, si por un lado la asignatura les resultó mayoritariamente muy buena, al consultar por su experiencia en la modalidad virtual el nivel de satisfacción disminuye. De este modo, observamos que un $42 \%$ califica la experiencia como "buena" mientras que un $28 \%$ la califica de "muy buena". Una de las personas participantes comenta:

"Me permite estudiar en cualquier momento y lugar. Teniendo en cuenta mi realidad laboral y familiar me ayuda mucho tener la posibilidad de estudiar en forma virtual."

Las respuestas no están tan concentradas en los niveles más altos de clasificación como en el caso de la pregunta sobre su conformidad respecto de la asignatura. Hay algo con la modalidad virtual que es valorado de manera negativa por un grupo de estudiantes. De este modo, observamos que el $10 \%$ sostiene que la experiencia de haber cursado de manera virtual fue entre mala y muy mala.

Entendemos que la disconformidad se relaciona principalmente con dos situaciones: a) quienes han tenido dificultades para acceder a conexiones de calidad, o debieron realizar sus estudios a través de soportes incómodos como puede ser un celular, b) quienes reclamaron que les hubiese gustado contar con más encuentros sincrónicos (por zoom, jitsi, meet u otra plataforma). En este sentido, corresponde 
aclarar que la UNLa cuenta con un campus virtual con el programa Moodle y que la mayor parte de las actividades propuestas han sido diacrónicas. Las actividades semanales consisten en la realización del siguiente recorrido: lectura de "guiones de clase", especialmente preparados para la enseñanza virtual. Además, lectura de bibliografía obligatoria, participación en un foro de discusión, mientras que la evaluación consiste en dos exámenes.

Se observa por un lado un alto grado de acuerdo con la cantidad de materiales y actividades propuestas (solo el 15\% sostiene que los materiales son excesivos). Asimismo, el $85 \%$ respondió que los materiales le resultaron interesantes, y el $66 \%$ sostiene que los textos vistos en clase no se han superpuesto con los textos trabajados en otras asignaturas, con lo cual podemos inferir que para la mayoría los temas y textos son novedosos.

Volviendo a la diferente valoración que dieron al curso y a la experiencia de haberlo realizado en la modalidad virtual, creemos que es importante tener en cuenta que la formación en y para los derechos humanos requiere construir escenarios de trabajo en los cuales sea posible el diálogo, el intercambio de opiniones, la deliberación y el debate. En nuestra experiencia en el aula presencial estos intercambios ocurren de manera cotidiana, y son parte de lo que se añora y se señala como un aspecto deficitario del curso. Así lo atestiguan testimonios como el que sigue:

"Al ser virtual falto el intercambio que existe en la presencialidad, para saber si estaba comprendiendo bien los temas".

"Considero que de forma presencial los temas se pueden ver mejor y se aprende un poco más".

Con relación a los aspectos positivos del curso, se valora en primer lugar la organización y modalidad del dictado del curso, siendo destacado por el 48 por ciento de las personas encuestadas. Se destacan los guiones de clases elaborados específicamente para el dictado virtual, así como las posibilidades que la modalidad virtual presenta, lo cual se encuentra vinculado con la segunda dimensión valorada, que es la posibilidad de organización autónoma durante este contexto excepcional. El $21.3 \%$ de las personas encuestadas valora esta dimensión. Se observan respuestas como las siguientes:

"Me permite estudiar en cualquier momento y lugar. Teniendo en cuenta mi realidad laboral y familiar me ayuda mucho tener la posibilidad de estudiar en forma virtual"

"Siento que el hecho de poder tener mi tiempo cada semana para leer bien todo y buscar más información sobre los temas para poder contestar los foros no es 
algo que hubiera hecho si fuera presencial. Quizás hubiera presenciado la clase con interés para después desconectarme del tema, pero tener todo en el aula para contestar los foros, y no tener que hacer un trabajo práctico grupal por obligación hizo todo más dinámico"

Las alternativas que ofrece la experiencia de estudiar en la modalidad virtual y por lo tanto administrar el tiempo de manera autónoma han sido altamente valoradas, ya que hasta el año 2019 la mayor parte de la enseñanza universitaria transcurría en entornos presenciales donde escaseaba la autonomía para la gestión del tiempo. Sin embargo, es pertinente mencionar que hay quienes opinan que la virtualidad permitió un manejo distinto de los tiempos y la posibilidad de compaginar y ordenar horarios, mientras que hay quienes consideran que el tiempo se volvió un factor limitante, debido a la complejidad de la organización de múltiples tareas y responsabilidades. Este dato resulta importante de considerar y amerita nuevas investigaciones para conocer los impactos desigualdades que puede presentar esta dimensión desde una mirada interseccional. Ha sido ampliamente señalado que los usos del tiempo son desiguales, por ejemplo, entre varones y mujeres, por lo que es importante ahondar en relevamientos que permitan adentrarse en las dificultades que se articulan al considerar el género, la inserción territorial, la edad, las condiciones socioeconómicas, entre otras variables.

En cuanto a la organización y la disposición para el estudio en entornos virtuales, surgen afirmaciones como las siguientes:

"Porque me da la libertad de leer, subir las actividades a la hora que yo me pueda acomodar"

"La cursada virtual en mi opinión fue muy buena ya que a pesar de no ser presencial los docentes siempre estuvieron a nuestra disposición para sacarnos cualquier tipo de duda y además no tuve ningún inconveniente en comprender los temas vistos $\mathrm{y}$ en entender los textos".

Sin embargo, también hubo obstáculos y limitaciones. En este sentido un estudio realizado por el Instituto Internacional de la UNESCO para la Educación Superior en América Latina y el Caribe (UNESCO IESALC), se señala que "no existen todavía datos disponibles acerca de cuáles son según los estudiantes los principales problemas a los que se enfrentan durante la pandemia" (2020, p. 15). Sin embargo, en el mismo estudio se destaca que en base a una encuesta sobre esta cuestión realizada a los directores de las cátedras UNESCO en todo el mundo se sabe que: 
Los resultados sugieren que, a escala global, las principales preocupaciones son el aislamiento social, las cuestiones financieras, la conectividad a internet y, en general, la situación de ansiedad relacionada con la pandemia. En Iberoamérica, sin embargo, el orden de las preocupaciones es algo distinto pues las cátedras UNESCO colocan, por encima de las demás, solo tres prioridades: la conectividad a internet, las cuestiones financieras y las dificultades para mantener un horario regular. (UNESCO IESALC, 2020, p. 16)

Podemos identificar algunas dimensiones a partir del análisis de las respuestas abiertas. Hemos identificado dificultades vinculadas a las siguientes dimensiones: el $83 \%$ de las personas encuestadas señala dificultades vinculadas a la modalidad virtual; el 13\% refiere limitaciones y dificultades vinculadas a la conectividad y/o acceso y uso de tecnologías; por último, un $4 \%$ expresa encontrar obstáculos relacionados con la organización del tiempo.

Dentro de la primera dimensión, el dato relevante, al que se hizo mención en el apartado anterior, es que el grupo de estudiantes vincula la presencialidad con el enriquecimiento de los debates e intercambios. Valoran la posibilidad de realización de encuentros sincrónicos como una forma de favorecer dicha instancia de diálogo e intercambio. No obstante, dentro de la misma dimensión hay quien señala que resulta una dificultad la lectura de los materiales bibliográficos.

En otros casos, la organización del tiempo resultó compleja y la presencia de largas horas frente a una computadora empeoró la situación, al tiempo que expresaron tensiones al tener que articular el estudio con el trabajo:

"Porque al trabajar online, se hace muy pesado pasar tantas horas frente a la pc "

"Difícil organizar los tiempos para estudiar, recordar que días se suben cada clase, hasta qué fecha y hora se pueden realizar los cuestionarios, etc. Demasiado tiempo frente a la computadora."

"La modalidad de clase a clase, entre los textos, el estudio y el trabajo, se hace muy difícil, al menos para mí, manejar los tiempos"

Respecto a la categoría que agrupa las respuestas que refieren dificultades de conectividad y/o acceso y uso de tecnologías, se observan referencias a la falta de internet vinculadas a las zonas de residencia, así como de manejo de la tecnología necesaria. Este es un dato de importancia, ya que como señala en un estudio conjunto entre la UNESCO y la CEPAL "Es preciso entender estas brechas desde una perspectiva multidimensional, porque no se trata solo de una diferencia de acceso a 
equipamiento, sino también del conjunto de habilidades que se requieren para poder aprovechar esta oportunidad, que son desiguales" (CEPAL- UNESCO, 2020, p. 7).

Si bien el área de campus virtual de la universidad brindó capacitaciones desde el inicio del dictado de la medida de aislamiento (y anteriormente también), para un grupo de estudiantes el manejo de los dispositivos y uso del aula pudo presentar dificultades, como se expresa en el siguiente testimonio:

"Me estresa mucho el hecho de no manejar bien los dispositivos, lo que no me permitió tener el tiempo suficiente en los parciales donde corría el tiempo para responder, y el tener un dispositivo (computadora lenta y antigua) y eventualmente problemas de conectividad".

Con relación a esta dimensión es importante destacar dos medidas llevadas a cabo para disminuir la brecha digital: la primera es la liberación del uso de datos en los sitios web de las universidades. Esta medida implicó que las compañías de telefonía celular permitieran a los estudiantes universitarios acceder de forma gratuita a las plataformas educativas y aulas virtuales que utilizan las universidades. Si bien no se ha recabado el impacto de esta medida en los estudiantes que han cursado el seminario en particular, podemos señalar que resulta una medida de carácter amplia que presupone una base para la democratización del acceso a servicios de internet con fines educativos. La segunda medida es que al inicio de la pandemia y ante el diagnóstico compartido de la existencia de dificultades vinculadas a la conectividad, la Universidad Nacional de Lanús implementó becas de conectividad. Las mismas fueron de carácter excepcional implementadas por el Programa Compromiso Educativo.

Hasta aquí hemos presentado una breve descripción de la vida estudiantil durante la pandemia. A continuación, quisiéramos adentrarnos en otra de las preguntas que orientan nuestra indagación: ¿Cómo perciben los y las estudiantes de la universidad a los derechos humanos?

\section{Percepciones sobre los derechos humanos}

En el siguiente apartado analizaremos las cuestiones referidas a las ideas preexistentes del grupo de estudiantes que participaron en las encuestas. Para ello les preguntamos: ¿El Seminario contribuyó a modificar tu percepción de los DDHH? Este interrogante surge de la consideración de que quienes transitan la educación universtaria ya atravesaron dos niveles obligatorios del sistema educativo, en los cuales la EDH ha tenido algún tipo de anclaje. Pero además, entendemos que la 
temática de los derechos humanos desde el retorno de la democracia tiene una presencia importante en la cultura política de la Argentina.

En la muestra, el 85\% sostuvo que modificó su antigua percepción sobre los derechos humanos, pero al mismo tiempo sostienen que no han recibido formación previa en derechos humanos (78\%), por lo cual es altamente probable que esta asignatura haya incidido de manera significativa en las ideas preexistentes. Por otro lado, quienes sí contaban con formación en derechos humanos comentan que la recibieron en el nivel superior (43\%) y en el nivel medio (39\%). Muy pocos casos mencionan haber recibido formación en derechos humanos en la escuela primaria.

Por otra parte, quienes entienden que su percepción sobre los derechos humanos no se vio modificada luego de haber cursado esta asignatura (15\%); la mitad afirma contar con formación previa en derechos humanos, y de ellos la mayoría proviene del nivel superior 34\% (la mayoría de la experiencia en otras materias de su carrera y en otras carreras de otras universidades) y esta constituye la principal justificación por la cual consideran que, luego de atravesar este curso, no perciben que haya cambiado su comprensión sobre los derechos humanos.

\section{Cambios en la forma de percibir a los derechos humanos}

A continuación, nos proponemos profundizar el análisis sobre el impacto que pudo haber tenido en quienes participaron de la encuesta el hecho de atravesar espacios formales y formativos sobre los derechos humanos.

Recuperando los instrumentos internacionales rectores en materia de educación en derechos humanos $(\mathrm{EDH})$, nos interesa destacar la definición realizada en las Directrices para la elaboración de planes nacionales de DDHH (1997; A/52/469/ Add.1) elaboradas por la Oficina del Alto Comisionado de las Naciones Unidas para los Derechos Humanos en el marco de las actividades del Decenio de las Naciones Unidas para la educación en la esfera de los derechos humanos (19952004). La misma sostiene que la promoción de los derechos humanos tiene lugar en el contexto de campañas educativas que abarcan tres dimensiones:

a) Conocimientos: actividades de información acerca de los derechos humanos y de los mecanismos existentes para su protección

b) Valores, creencias y actitudes: promoción de una cultura de derechos humanos mediante el fomento de valores, creencias y actitudes que sustenten esos derechos;

c) Adopción de medidas: fomentar la adopción de medidas para defender los derechos humanos y evitar las violaciones de esos derechos. (p. 6) 
En función de estas definiciones interpretamos las respuestas sobre la incorporación de información, de valores y de apelación a modificar aspectos conductuales, tanto en la vida cotidiana como en el futuro ejercicio de la profesión. Considerando este marco conceptual, las respuestas pueden ser interpretadas del modo que presentamos a continuación.

a) Aspectos informativos: son aquellas respuestas que explicitan la nueva información obtenida, que amplían la perspectiva y conocimientos, que otorgan nuevos "puntos de vista". Algunos ejemplos extraídos de las preguntas abiertas que refieren a esta categoría son:

"Porque yo no sabía lo inmenso que era este mundo de los ddhh y todo lo que abarca" o "Porque había mucho contenido del cual no vemos a menudo y estuvo bueno poder aprender."

b) Aspectos valorativos: Las respuestas enmarcadas en esta categoría son aquellas que denotan una valoración en términos positivos respecto de los derechos humanos. En este tipo de respuestas encontramos frases que aluden a que pudieron "tomar conciencia sobre situaciones de injusticia", "ver lo que está mal", la importancia de los ddhh, comprender la desigualdad, sensibilizar, "prestar atención" a problemáticas, aprender a ser empático, tener conciencia; desnaturalizar. Una argumentación típica del tipo valorativo se puede observar en frases como:

"Me permitió entender lo importante que son" o "Me permite abordar las temáticas desde otra perspectiva, con un lado mucho más humano".

c) Aspectos conductuales: hace alusión a la incorporación de herramientas para la defensa de los derechos humanos, posiciones proactivas respecto de su defensa tales como "hacerse escuchar", "hacerse respetar/ hacerlos respetar"; "hacerlos efectivos-efectivización de los ddhh", así como diferentes enseñanzas para aplicarlas en la vida cotidiana y/o en la profesión:

"Porque me permite ampliar mi perspectivo sobre el tema y poder contar con mayores herramientas a la hora de actuar en mi vida social y profesional"; "Me ayudó a conocer cuáles son los derechos de las personas y a hacer que se cumplan." o "Conocía los derechos humanos pero este seminario hizo que me dé cuenta de conductas persistentes tanto en mi como a mi alrededor que deben cambiarse."

Asimismo, existen respuestas que involucran dos o los tres aspectos al mismo tiempo, por lo cual se desagregaron las respuestas en una o más categorías de manera de no perder ninguna respuesta en el procesamiento de los datos. 
La mayoría de las respuestas rescata los aspectos informativos incorporados (77\%); mientras que en segundo orden de aparición encontramos justificaciones que aluden a los aspectos valorativos (13\%) y, por último, se encuentran enunciados referidos al comportamiento $(8 \%)$.

Solo 10 estudiantes aludieron a las tres dimensiones simultáneamente. Esto no significa que los demás estudiantes no modifiquen su visión en los sentidos no mencionados por ellos mismos, lo que significa es que el grupo de estudiantes que sí argumentaron en el triple sentido lo han manifestado como importante de rescatar (y de alguna manera lo han hecho presente en su justificación, es decir, lo han hecho de manera consciente). A continuación, algunos ejemplos de ello:

"Si, modificar y ampliar, aunque crea que los derechos humanos no se cumplan para gran parte de las personas a nivel mundial, es importantísimo abrir tu cabeza, reflexionar, mirar un video y decir yo hacía eso o yo pensaba eso y tratar de cambiar, tratar de ser mejor persona, o de intentar poder hacerle ver a la gente que tenemos alrededor que está mal lo que les enseñaron, que debemos de deconstruir para construirnos de manera unida y solidaria para con el otro."

"Siempre tuvo una importancia para mí, pero ahora me doy cuenta de muchas acciones $o$ actitudes que todo el mundo tiene que cambiar para poder mejorar, me dio una base más fuerte para defender a quienes no les otorgan estos derechos."

\section{Algunas consideraciones acerca de la importancia de reflexionar sobre un tema (re) conocido:}

Respecto de las ideas preexistentes, algunos autores del campo de la EDH sostienen que la mirada que existe en nuestra sociedad en materia de derechos humanos sigue siendo aún muy restringida, lo cual obedece generalmente a razones históricas, y que la percepción del imaginario colectivo sobre los derechos humanos está demasiado focalizada en la desaparición forzada, la tortura, las ejecuciones extrajudiciales, lo cual vuelve invisibles otros contenidos, como los derechos económicos y sociales (Salvioli, 2014).

Hemos observado una tendencia a asociar derechos humanos con el período de la última dictadura militar argentina, ignorando otras problemáticas como por ejemplo las desigualdades en el acceso a la salud o a la educación y las tecnologías. Por ello, las nuevas informaciones, conocimientos y saberes académicos aprendidos son elementos que se reconocen como movilizadores y modificadores de su imaginario preexistente (77\%). Recordemos que el programa de la materia recorre 5 ejes fundamentales (formación histórica de los ddhh, proceso de memoria verdad y 
justicia, género, cultura y migraciones). Esta situación, de aparente sencillez, como la de incorporar a las ideas del sentido común información académica, implica una profunda transformación no solo del contenido de esta, sino que también del modo de procesamiento y la estructuración de la misma. Unas frases ilustran de forma elocuente este proceso:

"Tenía una respuesta muy corta sobre el tema, pensaba que solo era un pensamiento que solo le pasa a los de la izquierda y sobre la dictadura y es un tema tan amplio y es por el simple hecho de ser una persona con derechos."

"Si, siempre se escucha una frase horrible como "los derechos humanos son para los chorros" (los ladrones) o "siempre robando con los derechos humanos". Esas frases (nefastas) insertadas en la sociedad promueven una imagen negativa acerca de los derechos humanos. Abordar las problemáticas desde un enfoque de derechos promueve sociedades más justas e inclusivas."

En el momento en que remitimos que hemos hablado desde prejuicios y/o estereotipos sobre los derechos humanos, se está ilustrando que, en las ideas que se tiene respecto a un objeto, no solo se juegan elementos psíquicos (como el pensamiento, la actitud o una imagen representativa que se elabora en el marco del pensamiento) sino que estamos hablando de ideas compartidas con otros, es decir, ideas socialmente elaboradas donde no se habla en términos particulares sino que se habla en términos sociales, ya que lo que se alude allí no se ha conformado por fuera de estructuras sociales, de elementos ideológicos, culturales, de comunicación social, etc.

Los derechos humanos son percibidos de forma naturalizada, como si se tratase de un objeto con propiedades tautológicas, tal cual describen las siguientes afirmaciones extraídas de los estudiantes: "cosas que ya se tienen por sabidas en obviedad" o "que no hay que dar por sentado la percepción que cada individuo tiene de los derechos humanos". Es decir que hay un reconocimiento explícito de que se tenía una idea y a la misma vez "no se tenía idea" de lo que son los ddhh. Esta reflexión fue posible por la incidencia de la asignatura aquí analizada (recordemos: el seminario de justicia y derechos humanos), tal como describe otro estudiante

"Me hizo pensar en que no hay que dar por sentado la percepción que cada individuo tiene de los derechos humanos y lo importante que es reforzar el conocimiento de los mismos, conocerlos es el primer paso para hacerlos cumplir".

Tal vez esto sea porque el término Derechos Humanos lleve en su propia relación fundada en el carácter lineal de la lengua, lo que Saussure llama sintagmas, donde las unidades contraen relaciones sintagmáticas, que son relaciones "en presencia". 
En este sentido, por ejemplo, es esperable que todas aquellas ideas que involucran a sujetos no humanos, como los animales, el ambiente, los recursos naturales no formen parte de la representación social en cuestión, ya que las cuestiones referidas a la última dictadura militar se erigieron históricamente en la Argentina como hegemónicas.

Por otro lado, encontramos en los relatos la idea de que los derechos humanos son "algo que uno asume como logrado", es decir, que además de ser un concepto en apariencia obvio (no más complejo de lo que sus términos explican) aparece como un estado actual de la sociedad, un capítulo cerrado de la historia reciente y/o un "triunfo" en la misma. En otras palabras, aparentemente, se lo construye como un objeto cerrado y estático, totalmente contrario a su realidad, ya que es un concepto esencialmente dinámico y abierto. Los derechos humanos aparecen a lo largo de la historia de los pueblos como conquistas político-sociales que los colectivos han logrado mediante el reconocimiento de estas en sus ordenamientos jurídicos.

Finalmente, creemos que incidir sobre ese conjunto de ideas del imaginario social sobre el objeto, es incidir sobre los aspectos simbólicos de los derechos humanos, lo cual colabora sin lugar a dudas con una efectiva ( $\mathrm{y}$ tan esperada) difusión del paradigma de los derechos humanos y con la construcción de una cultura afín a ellos, al menos en el ámbito discursivo-reflexivo de los estudiantes.

"No era consciente de varias situaciones injustas en nuestra sociedad que a mi parecer son bastante naturalizadas y que debemos conocer para poder revertir."

De esta manera, el conocimiento se torna en potencia movilizadora de transformación social. La posibilidad que brinda el hecho de reconocer una situación y, en función de ese saber, la posibilidad de transformarlo. Es decir, la educación en derechos humanos como la formación de agentes de argumentación (Rodino, 2014). Para la autora, la EDH logra más que capacitarlos en derechos humanos porque los ayuda a ver los derechos como una "vara de medir" su trabajo regular -es decir, como lo que son, parámetros éticos, críticos y políticos-; para identificar los problemas sociales que enfrentan a diario; para ubicar su propio rol frente a ellos (real y deseable); y para visualizar correctivos que está a su alcance impulsar. Los compromete ética y políticamente a movilizarse para producir cambios. (Rodino, 2014, p. 132)

\section{A modo de conclusión}

Las y los estudiantes no eligieron ser estudiantes en entornos virtuales y, según sus testimonios, no estaban del todo preparados para ello cuando en marzo del 2020 la 
Humanidad debió recluirse. Ser estudiante viviendo en pandemia está siendo una experiencia con ventajas y desventajas, pero, definitivamente, con aprendizajes. De un lado nos topamos con los aprendizajes ligados a aquello que podemos denominar "alfabetización en entornos virtuales", tal como relatan los testimonios fue necesario aprender a ser estudiante a distancia, pero al mismo tiempo lidiar con las angustias propias de estos tiempos de incertidumbre. Las desigualdades se profundizan y las condiciones en las que se estudia, se administra el tiempo y al mismo tiempo se realizan otras tareas vitales (como el trabajo remunerado o no - en particular las tareas de cuidado- por nombrar dos que fueron mencionadas en las encuestas) muestran realidades muy heterogéneas, tal como lo es la población universitaria en la Argentina.

En este contexto, el contenido de lo que se enseña y se aprende en el espacio curricular que hemos analizado en este estudio, es decir: los derechos humanos, cobra un sentido particular en este duro contexto. Es posible identificar escenas de desigualdad y desesperanza, como cuando nos escriben para avisar que no podrán "asistir" a clase sincrónica por estar atravesando (en primera persona o a través de una persona cercana) la enfermedad de covid-19. Las entregas de trabajos que se atrasan, las dificultades para realizar lecturas concentradas emergen, la falta de dispositivos adecuados se pone en evidencia... y, al mismo tiempo, la posibilidad de administrar de forma autónoma el tiempo y evitar los largos desplazamientos característicos de una gran localidad como lo es la Provincia de Buenos Aires se valora positivamente.

Tal como dijimos, nos topamos con valoraciones positivas y negativas respecto de la vida estudiantil en pandemia. Sin embargo, en cuanto a la enseñanza de los derechos humanos, podemos agregar que siete de cada diez estudiantes sostienen que haber realizado un curso sobre derechos humanos les ayudó a identificar nuevos temas y problemas sociales relacionados con su campo profesional, es decir, se reconoce la importancia del paradigma de los derechos humanos en su formación profesional, más allá de su condición de ciudadanía, que conoce y ejerce (o reclama) sus derechos. La fortaleza del paradigma está en transformar sus prácticas técnico-profesionales al modificar la comprensión sobre su campo profesional.

La formación en ddhh no solo es imprescindible para la formación de la ciudadanía, sino que es imprescindible para la formación de profesionales con compromiso con las causas sociales que atraviesan su campo, muchos de los cuales formarán -o forman- parte de los equipos técnicos de las áreas estatales que afectan directamente el goce de los derechos humanos de todas las personas. 
Tal cual lo enuncia Salvioli "la falta de disfrute de los derechos humanos dentro de una sociedad tiene una relación directa con la ausencia o insuficiencia de capacitación y educación en y para los derechos humanos en general, y particularmente, con la carencia de formación en derechos humanos a nivel universitario" (Salvioli, 2014 , p. 214). Pues la interacción con la información, los valores y las acciones vinculadas a la defensa de los derechos humanos ofrece argumentaciones y modos de re pensarse como personas ciudadanas y profesionales.

En este sentido:

La decisión política de implementar educación en derechos humanos, en la educación universitaria, pienso que no es una opción que dependa de una ideología determinada, es una decisión que expresa coherencia con una visión del mundo que respeta la dignidad y la libertad de todas las personas en condiciones de igualdad en todos lados. Educar a los graduados universitarios en derechos humanos supone contribuir a que el respeto de los derechos humanos no quede sólo en el discurso, sino que devenga un hacer. Yo creo que se trata de una política académica específica que se propone como objetivo explícito un cambio de cultura, ella debe además generar ciudadanía, conciencia de género, conciencia de derechos económicos, sociales y culturales tan importantes como los civiles y políticos, derechos del medio ambiente de la pluriculturalidad y de participación política. (Pinto,2021, p.202).

Frente a la necesidad de educación en derechos humanos en toda la trayectoria formativa, acompaña esta premisa la opinión de un grupo de estudiantes que también se manifiestan en este sentido; un $80 \%$ está de acuerdo en que todas las carreras universitarias deberían contar con EDH. Es decir, que no se trata de una aspiración por parte de los grupos y órganos de expertos en la materia, el hecho de que los ddhh sean un contenido formativo básico, sino que también se cuenta con la adhesión por parte de quienes lo cursan, pues reconocen la necesidad en este sentido, una vez concluido el curso, ciertamente.

Sin embargo, a la vista de los grandes cambios que produjo la pandemia en nuestras cotidianidades, es necesario replantear las formas y metodologías disponibles a través de medios virtuales para la enseñanza y el aprendizaje de estos contenidos. Necesitamos conocer y desplegar recursos más eficaces en tiempos de pandemia, pues estos saberes no se circunscriben a lo informativo, tal como se señaló anteriormente.

Trabajar por la sensibilización, la empatía y la implementación de instancias de deliberación e intercambios que permitan argumentar en profundidad -y en complejidad- sobre los debates de este campo problemático, son algunos de los desafíos que quedan pendientes. Pues el hecho de garantizar el respeto a los derechos 
humanos solamente por medio de la ley no es suficiente para que se asimile una auténtica comprensión y ejercicio de estos.

Es imperativo pensar cómo hacer para que los sujetos se comprendan como humanos y traten a los otros desde esa misma perspectiva, no solo por la necesidad de reconocer los derechos sino también de hacerlos prácticos y vincularlos a lo que hoy en día entendemos o deberíamos entender por humanidad" (Quintero Mejía y Camargo, 2010, p. 45). Es por ello que sostenemos que la práctica pedagógica debe estar atravesada por los significados, los sentidos y las reflexiones acerca de cómo construir una humanidad más humanizada, sensible a los dolores y activa en la lucha a favor de un mundo más justo, en el cual no solo sean realmente posibles los derechos humanos, sino que sean una realidad efectiva para todas las personas.

\section{Referencias}

CEPAL- UNESCO (2020). La educación en tiempos de la pandemia de COVID-19. https:// repositorio.cepal.org/bitstream/handle/11362/45904/1/S2000510_es.pdf

Pinto, M. (2020), La alfabetización en derechos humanos en la Universidad de Buenos Aires, en Badano R. y Cruz, V (comps.), Conversaciones en plural. Educación superior, derechos humanos y desigualdad en tiempos de pandemia, Editorial de la Universidad Nacional de La Plata (EDULP).

Quintero Mejía, M. y Camargo, M. (Comps.) (2010). Educación en derechos humanos. Perspectivas metodológicas, pedagógicas y didácticas. Universidad de La Salle-Colombia

Resolución 59/113 de la Aasamblea General de las Naciones Unidos "Programa Mundial de Educación en Derechos Humanos", A/RES/59/113 (10 de diciembre de 2004). https://www.ohchr.org/sp/issues/education/educationtraining/pages/programme. aspx\#: :text=El $\% 2010 \% 20 \mathrm{de} \% 20$ diciembre $\% 20$ de,humanos $\% 20$ en $\% 20$ todos $\% 20$ $\operatorname{los} \% 20$ sectores.

Rodino, A. M. (2014). Pensar la educación en derechos humanos como política pública. Revista de ciencias sociales, segunda época $N .^{\circ}$ 25, 129-139. Buenos Aires: Universidad Nacional de Quilmes. http://www.unq.edu.ar/advf/documentos/5939558c6ba3b.pdf

Salvioli, F (2014). Educación superior en derechos humanos. Una herramienta para la organización y el desarrollo de la política pública del Estado. Revista de ciencias sociales, segunda época $121 N^{\circ}$ 25, 121-12. Buenos Aires: Universidad Nacional de Quilmes. https://ridaa.unq.edu.ar/bitstream/handle/20.500.11807/1593/09_RCS-25_dossier8. pdf? sequence $=1$ \&isAllowed $=\mathrm{y}$

UNESCO- IESALC. (2020). Informe COVID-19 y educación superior: De los efectos inmediatos al día después. Análisis de impactos, respuestas políticas y recomendaciones. http://www.iesalc.unesco.org/wp-content/uploads/2020/05/COVID-19-ES-130520.pdf $118 \begin{aligned} & \text { Revista Latinoamericana de Derechos Humanos } \\ & \text { Volumen 33 (1), I Semestre 2022 } \\ & \text { ISSN: } 1659-4304 \cdot \text { EISSN: 2215-4221 }\end{aligned}$ 